\title{
Una propuesta orientada al alcance de logros académicos y a la permanencia del estudiantado de sétimo año en la educación secundaria
}

\section{Proposal for Academic Achievement and Permanence of Students in the $7^{\text {th }}$ Grade ${ }^{1}$}

\author{
Luis Alfredo Miranda Calderón ${ }^{2}$ \\ División de Educación Básica, Centro de Investigación y Docencia en Educación \\ Universidad Nacional de Costa Rica \\ Heredia, Costa Rica \\ alfremira@gmail.com \\ Nury Ginnette Bonilla Ugalde 3 \\ Dirección de Tecnología Educativa \\ Universidad Técnica Nacional \\ Alajuela, Costa Rica \\ nury.bonilla@gmail.com
}

Recibido 05 de mayo de $2010 \bullet$ Aceptado 31 de agosto de 2010

Resumen. El presente artículo analiza el impacto de los procesos de cambio que grandes poblaciones de estudiantes experimentan al terminar la etapa de educación primaria e iniciar la educación secundaria. A partir de la investigación desarrollada se describen situaciones y aspectos que pueden determinar condicionantes para la adaptación en este nivel: cambios espacio-temporales, organizativos y dinámicos que configurarían el nuevo entorno educativo y contexto social en el que se desarrolla esta etapa. Se exponen los condicionantes que inciden en el aprendizaje del estudiantado que ingresa: programas, metodología para la enseñanza, estilos de aprendizaje y nuevas formas evaluativas. Como producto de los hallazgos de la misma investigación, se plantea una propuesta para atender la transición de la educación primaria a la secundaria. Dicha propuesta se conforma por un conjunto de lineamientos orientadores, dirigidos a la sensibilización y fortalecimiento de la mediación pedagógica para propiciar la permanencia, en el sistema educativo, del estudiantado proveniente de todo tipo de instituciones de educación primaria.

Palabras claves. Propuesta educativa, investigación, transición, educación primaria, educación secundaria.

$1 \quad$ [Translator's note: The Costa Rican education system is composed of primary education $\left(1^{\text {st }}-6^{\text {th }}\right.$ grade $)$ and secondary education $\left(7^{\text {th }}-11^{\text {th }}\right.$ grade).]

2 Académico en la División de Educación Básica del Centro de Investigación y Docencia en Educación de la Universidad Nacional, Costa Rica. Magíster con especialidades en Administración Educativa, Universidad de Costa Rica y en Formación de Formadores de Docentes de Educación Primaria, Universidad Nacional. Bachiller en Artes Dramáticas. Bachiller en Ciencias de la Educación en I y II ciclos. Licenciado en Ciencias de la Educación en I y II ciclos con énfasis en Educación Rural. Como maestro rural unidocente ha realizado diversas investigaciones y exploraciones relacionadas con el aprendizaje mediado por el uso y aprovechamiento del entorno natural. Maestro en la Escuela Nueva Laboratorio de la Universidad de Costa Rica, donde ha desarrollado procesos pedagógicos haciendo uso del juego escénico como una herramienta para el aprendizaje.

3 Máster en Psicopedagogía, Universidad Estatal a Distancia, Costa Rica y en Formación de Formadores de Docentes de Educación Primaria, Universidad Nacional, Costa Rica. En la actualidad dirige el Departamento de Producción de Materiales Didácticos en la UTN, del Centro de Formación Pedagógica y Tecnología Educativa. 
URL: http://www.una.ac.cr/educare

Abstract. This paper analyzes the impact of change processes experienced by many student populations when completing primary education $\left(1^{\text {st }}-6^{\text {th }}\right.$ grade) and starting secondary education $\left(7^{\text {th }}-11^{\text {th }}\right.$ grade). Based on the research conducted, this paper describes situations and aspects that may result in conditional factors for the student's adjustment at this level: time-space changes, as well as organizational and dynamic changes that would set the new educational environment and social context in which this new stage will be developed. Such conditional factors that affect learning in incoming students: programs, teaching methodology, learning styles and new evaluation methods will be discussed. As a result of this research, a proposal is presented to facilitate transition from primary to the secondary education. This proposal includes guidelines for awareness and strengthening of pedagogical mediation, which would contribute to the permanence of students from all types of institutions in the education system.

Keywords. Educational proposal, research, transition, primary education, secondary education.

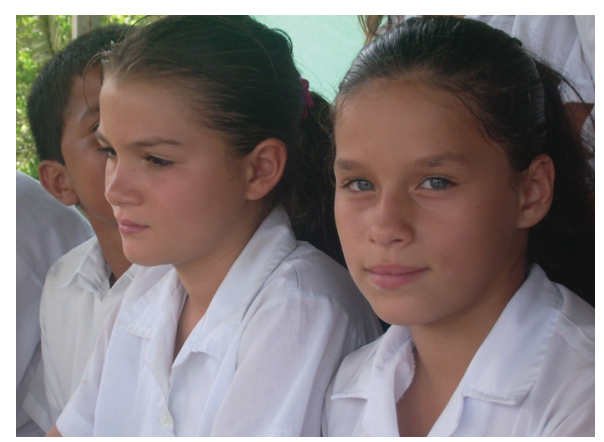

Es imprescindible concebir a las niñas, niños y adolescentes que ingresan al colegio, como personas en formación, con diversas situaciones emocionales debidas a los cambios propios de la adolescencia y sus implicaciones en la autonomía y autoestima.

Figura 1. Propiedad del autor.

\section{El estado de la cuestión}

La transición de la educación primaria a la educación secundaria en el contexto educativo costarricense constituye una etapa en la que grandes poblaciones de jóvenes estudiantes desertan del sistema educativo, con lo cual se condenan a la exclusión como destino inmediato.

Producto de una serie de transformaciones de la sociedad costarricense, esta situación constituye un serio desafío del sistema educativo, ya que, según las proyecciones, se compromete, a mediano y a largo plazo, el perfil ocupacional y profesional de las futuras generaciones de la sociedad costarricense. Una problemática compleja que obedece a diversos factores y que ha sido objeto de análisis desde diversas instancias que atienden la educación pública.

La concepción de la premisa de esta investigación surge a partir de la situación problemática que se aprecia durante los últimos ocho años en el marco del Sistema Educativo Nacional, caracterizada por los altos índices de deserción, ausentismo y baja promoción en el primer nivel de la educación secundaria. Esta etapa de transición de la educación primaria a la secundaria constituye quizás el periodo más frágil y vulnerable, si se considera que es en el que grandes poblaciones de jóvenes estudiantes desertan del sistema educativo condenándose a exclusión. La mayor acción desplegada en él va a generar menos insatisfacción, mayor conciencia de los estudiantes por lo que 
están haciendo, mejor rendimiento académico y, sobre todo, reducción del fracaso escolar. Según el Informe 2011 del Estado de la Educación:

(...) El séptimo año se mantiene como el momento más problemático de abandono del sistema educativo (...) el problema y sus causas se mantienen, y los avances, aunque importantes, todavía no alcanzan. Se requiere entonces redoblar esfuerzos, poniendo especial interés en ciertos tipos de colegios y zonas del país, así como en los mecanismos de seguimiento a los estudiantes más vulnerables. (...) En números absolutos, entre 1999 y 2009 un promedio de 35.765 alumnos por año fue expulsado del sistema educativo para un total de 393.412 estudiantes, a los que no es posible darles seguimiento (...). (Programa Estado de la Nación, 2011, pp. 124-125)

Estos datos constituyen un llamado de atención sobre la necesidad de mantener, profundizar y afinar las estrategias de retención, tanto por niveles, como por regiones y tipos de centros educativos.

Otro antecedente que ilustra la realidad que experimenta la población del $\mathrm{II}^{\circ}$ y III $^{\circ}$ ciclos de las instituciones educativas, constituye el hecho de enfrentarse luego de seis años de educación primaria, en el mejor de los casos, a una educación secundaria que le demanda, sin procesos de inducción o transición alguna, el cumplimiento de múltiples responsabilidades académicas.

En el caso específico de los estudiantes y las estudiantes de escuelas rurales, durante su formación escolar han sido orientados por una o un solo docente que imparte la totalidad de materias a todo el alumnado que asiste al plantel escolar. Sin embargo, al ingresar al colegio, una serie de cambios afectan su rendimiento: la diversidad de asignaturas, varios profesores y profesores que las imparten, consumo de extensos períodos de tiempo en el traslado a los centros de estudios, acceso y uso de recursos bibliográficos, alimentación y carencia de hábitos y técnicas de estudio. Todo lo cual agrava aún más un proceso que debería ser llevado a cabo en forma particular, si se toma en cuenta la condición de vulnerabilidad que sufre la educación secundaria, como lo plantean Guendel, Osorio y González (1999):

En el tercer ciclo de la Educación Básica, donde el sistema educativo muestra con mayor claridad su falta de calidad y su ineficiencia. Es también en estos niveles donde es posible constatar la estrecha relación de la deserción en la educación con el trabajo infantil, la explotación sexual, la drogadicción y, en general, con el riesgo de la niñez y la adolescencia. Es en el primer año de la secundaria cuando comienza la ruta crítica de la salida del sistema educativo y la incorporación al trabajo infantil y a otras actividades que constituyen la antesala de situaciones o de conductas que derivan en conflicto con la ley o que conculcan, como el trabajo y la explotación sexual infantil, las posibilidades de desarrollo pleno de las personas adolescentes y la construcción de un mejor futuro. (p. 48)

Esta condición contrasta dramáticamente con la de otros jóvenes provenientes de comunidades cercanas y centros escolares que brindan a sus estudiantes mayor diversidad de asignaturas y docentes a cargo. La acentuación de las desigualdades y la exclusión se tornan más evidentes, si se 
considera que la mayoría de estas jóvenes y estos jóvenes pierden gran cantidad de lecciones, lo que suma más dificultades a su condición. Esto se refleja claramente en los resultados de la Primera Encuesta Nacional de Juventud Costa Rica (Consejo Nacional de Política Pública de la Persona Joven, 2008) donde se afirma:

El panorama de las personas jóvenes en el rango de edad de 15 a 17 años es de central importancia desde el punto de vista del derecho de la educación, pues se registra una ruptura en el tránsito de la educación primaria a la secundaria, así como la retirada progresiva del sistema educativo durante la secundaria. Los factores estudiados muestran algunos de los indicadores más sensibles sobre calidad de la educación (incluyendo posiblemente las estrategias didácticas), restricciones estructurales en cuanto a cobertura y acceso, así como las dificultades intrínsecas al sistema (...). (p. 45)

En realidad el problema no se centra en la mediación pedagógica de las escuelas rurales, sino que se agrava en el primer año de la secundaria, que no considera el modelo pedagógico de estas escuelas de las cuales procede un alto porcentaje de sus estudiantes, ni establece criterios pedagógicos para atender la población proveniente de este tipo de instituciones educativas. Los resultados en la promoción e índices de deserción así lo demuestran, con el agravante de incidir mayormente en la población mencionada y que paradójicamente debiera ser atendida como prioridad, si se considera la necesidad de fortalecer equitativamente la educación de los habitantes de zonas rurales, como mecanismo indispensable para el desarrollo de sus comunidades.

Los niños y las niñas que ingresan al colegio provienen de instituciones educativas de educación primaria, las cuales contrastan radicalmente con el modelo educativo prevaleciente en la secundaria, caracterizado por una serie de factores como lo describe Alfaro et al. (2008):

El sétimo año de la educación formal se da en el marco de cambios profundos y drásticos en la población estudiantil de este nivel, en la que algunos o algunas ya están adentrados en la adolescencia y otros u otras se encuentran en el paso de la niñez a la adolescencia. En este proceso, se les atribuyen inseguridades, indefiniciones, carencias, identidad ambigua y necesidad de asentamiento interior y social con múltiples manifestaciones que consumen gran parte de las capacidades del sujeto. No obstante, también son audaces, desafiantes, con posibilidades de diversificar su atención en múltiples intereses y pueden realizar varias actividades simultáneamente. (p. 45)

Otro aspecto que requiere su debido estudio es el de la edad como factor determinante en el ingreso a la educación secundaria, etapa caracterizada por notables cambios en el desarrollo físico y afectivo de esta población. Al ingresar al colegio no dejan de ser niños y niñas debido al hecho de acceder a otro tipo de institución educativa. Sus inquietudes y necesidades merecen ser atendidas en este momento tan particular y la mediación pedagógica debe considerar seriamente este asunto, ya que al coartar la necesidad expresiva y lúdica de los niños y niñas, se transgrede, de diversas formas, el ejercicio de los derechos vinculados con la educación y el desarrollo de las potencialidades de los mismos. 


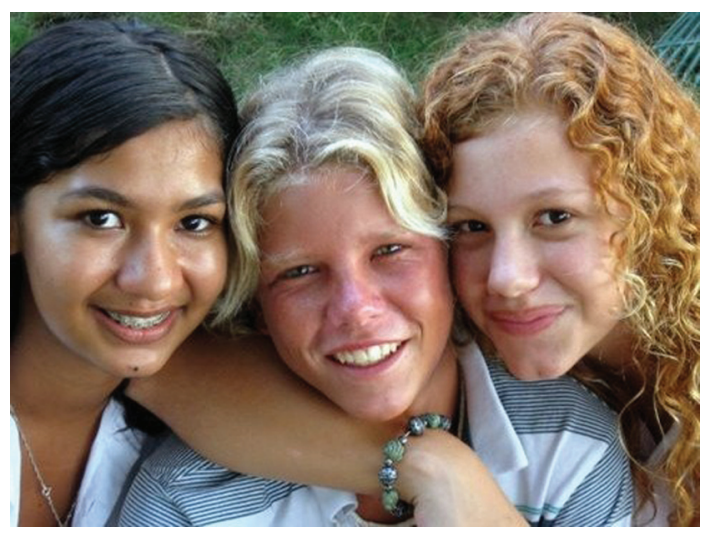

Es necesario precisar acciones orientadas a que la educación secundaria fomente el desarrollo pleno de las personas, la equidad e igualdad, el respeto a la diversidad, la construcción del conocimiento y de la acción basado en los derechos humanos.

Figura 2. Propiedad del autor.

En este marco de exclusión, los resultados de los diagnósticos e investigaciones desarrolladas muestran cifras alarmantes en términos de deserción y repitencia de estudiantes provenientes de estas instituciones, lo cual se agrava en los niveles iniciales de la educación secundaria. En este sentido, los resultados académicos de las instituciones educativas no deben ser asociados exclusivamente a variables de orden institucional sino, además, a las particularidades geográficas, económicas, sociales y culturales que caracterizan cada espacio y región del país, y que, desafortunadamente, no han sido consideradas en la formulación de políticas educativas.

Tales divergencias inciden dramáticamente en la apertura y cierre de cada curso lectivo, como se observa todos los años en relación con las rutas de transporte de los colegios de comunidades rurales, la contratación de docentes que atienden los procesos educacionales de las instituciones de primaria y secundaria, un calendario escolar descontextualizado de las actividades y épocas de producción en las que participan gran cantidad de jóvenes de contextos rurales y no rurales, y que, en muchos casos, constituye un factor detonante de deserción ante ofertas de trabajo que les concede su entorno inmediato. Al respecto, Hernández, León, Ruiz y Zúñiga (2002) mencionan:

Las desigualdades en educación son especialmente notorias entre las zonas rurales y urbanas. Mientras en las áreas urbanas un $75 \%$ de los estudiantes continúa sus estudios en el nivel secundario, en las rurales solamente lo hace el 30\%; la realización de la Educación Diversificada y de la Educación Superior es un privilegio del 35\% de los jóvenes de zonas urbanas, y solo del $11 \%$ de los de zonas rurales. (p. 226)

En el caso de los jóvenes y las jóvenes que finalizan la educación primaria y, en particular de los adolescentes y las adolescentes de todas las zonas del país, es necesario analizar los constantes riesgos y amenazas a que se enfrentan, así como el modo en que inciden en su desarrollo y condicionan las oportunidades de aprendizaje.

Lo anterior obliga a precisar acciones orientadas a que la educación secundaria fomente el desarrollo pleno de las personas, la equidad e igualdad, el respeto a la diversidad, la construcción del conocimiento y de la acción basado en los derechos humanos. Además, este derecho a la educación supone la necesidad de desarrollar mecanismos que garanticen la permanencia de modalidades educativas, que van más allá de la escolarización formal, para abarcar el amplio rango 
de experiencias de vida y procesos de enseñanza y aprendizaje, que permiten a los niños y niñas, individual y colectivamente, desarrollar sus personalidades, talentos y habilidades de forma plena.

Por todo lo anteriormente expuesto, se evidencia la necesidad de vincular las decisiones educativas con una oferta académica basada en la realidad vivida por las instituciones educativas. Por lo tanto, las escuelas y colegios deben constituirse, en la medida de sus posibilidades, en instituciones que beneficien a la comunidad mediante la concepción y ejecución de proyectos educativos innovadores que potencien el desarrollo humano y anticipen, mediante la acción educativa, respuestas eficaces a problemas futuros. Para esto se requiere adaptar los modelos de trabajo de estas instituciones para que se ajusten a las características y condiciones de las comunidades donde están insertas.

Al procurar visualizar los obstáculos que deben hacer frente los niños y niñas que acceden a la secundaria, es necesario destacar algunos aspectos que influyen especialmente en la transición entre ambas modalidades y afectan su desempeño académico. Algunos de estos aspectos son los siguientes:

- La edad y desarrollo de los estudiantes y las estudiantes.

- El cambio del entorno educativo y la masificación del estudiantado.

- La diversidad de asignaturas y mayor número de docentes.

- Aumento de responsabilidades y deberes académicos, así como la adaptación a nuevas y diversas metodologías de enseñanza.

- El nuevo entorno social y las nuevas relaciones interpersonales.

- Mayor rigidez de las normas y grados de exigencia académica.

- La dispersión e intensificación de horarios.

- Menor apoyo del grupo familiar.

Se comprende que aspectos, como los anteriormente descritos, constituyen condiciones comunes y en muchos casos inevitables; pero en cuanto a políticas educativas, resulta primordial la urgencia de valorar lo que sucede o no sucede en esta etapa decisiva, y consolidar propuestas educativas de mayor calado y eficacia.

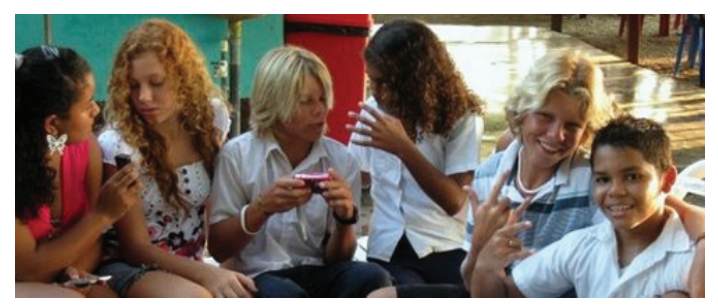

Figura 3. Propiedad del autor.
En el caso del estudiantado que finaliza la educación primaria y, en particular la adolescencia de todas las zonas del país, es necesario analizar los riesgos y amenazas a que se enfrentan, su incidencia en el desarrollo y condicionamiento en las oportunidades de aprendizaje.

\section{De la primaria a la secundaria}

Los niños y niñas provenientes de instituciones de la educación primaria que ingresan a los centros de secundaria provienen de una estructura basada en la mediación pedagógica personalizada, 
lo que contrasta dramáticamente con la del colegio, basada en secciones y en departamentos por asignaturas. Lo cual implica que el tratamiento pedagógico que aplique el personal docente debe considerar estos factores, tal como Alfaro et. al. (2008) describe en el Perfil Integrado Emergente de Desempeño Docente para Sétimo Año:

Promuevan espacios de aprendizajes significativos por medio de diversos métodos y técnicas, en los que se propicie la colaboración, la construcción de conceptos, la elaboración de trabajos, así como el intertransformación de ideas. Para ello, deben seleccionar las secuencias de actividades acordes con cada estudiante, el grupo, el tema por desarrollar, los contenidos por abordar, los objetivos propuestos y el contexto. Además, deben tener en cuenta para las características transicionales personales y sociales del estudiantado de sétimo año, la cantidad de estudiantes que conforman cada grupo, y las condiciones y medios para propiciar dichos aprendizajes. (p. 86)

Lo anterior muestra una serie de condiciones caracterizadas, en gran medida, por un tipo de metodología más centrada en el estudiantado y con una mediación más personalizada que no solo promueve, desarrolla y fortalece principios, valores y actitudes, sino que le permita al educando vivenciarlos en los diferentes ámbitos de su vida personal y social. Todo ello resulta dramáticamente contrastante con las asignaturas básicas y complementarias que caracterizan el III Ciclo de la Enseñanza General Básica.

\section{El estudio y su metodología}

El estudio se desarrolló en la modalidad de investigación cualitativa y en el marco de la maestría en "Formación de Formadores de Docentes de Educación Primaria" durante 2009 y 2010, en la cual se planteó, como objetivo inicial, promover un mejor desempeño académico del estudiantado proveniente de las escuelas rurales, como una forma de propiciar su permanencia en el sétimo año de la educación secundaria. Sin embargo conforme se avanzó en la investigación se determinó que la problemática no solo afectaba a la población mencionada, sino que se extendía además al estudiantado procedente de escuelas de zonas urbanas y semiurbanas.

El estudio destacó, como aspecto primordial, el análisis del grado de motivación que tienen los estudiantes de sétimo año durante los procesos de aprendizaje y los efectos en el desempeño académico al ingresar al nivel de la educación secundaria, por lo que se determinaron e interpretaron fenómenos y comportamientos que intervienen en los objetos de estudio de la investigación. Se pretende así, analizar y hacer procesos de reflexión que inviten a meditar sobre los procesos de transición y a establecer acciones concretas que busquen prevenir y atenuar la deserción. Al respecto, la sensibilización y la mediación pedagógica serán vitales para la permanencia en el sistema educativo de los estudiantes y las estudiantes que inician la educación secundaria.

\section{Actores directos e indirectos de la investigación}

El grupo de sujetos participantes que aportaron información y testimonios para el presente estudio estuvo constituido por 20 estudiantes de sétimo año que provenían de escuelas rurales y 
10 estudiantes que, por diversas razones, no han concluido el sétimo año. Además, participaron dos docentes de sétimo año y dos directores de instituciones de secundaria. La información que aportaron se recopiló utilizando las técnicas: entrevistas, grupos focales, cuestionarios, observaciones no participantes y análisis documental.

Se consideran, también, actores indirectos en esta investigación, a todas aquellas personas, poblaciones e instituciones vinculadas al quehacer educativo en los niveles $\mathrm{VI}^{\circ}$ y $\mathrm{VII}^{\circ}$ de la Enseñanza General Básica, tal es el caso de:

- Estudiantes del último nivel de la educación primaria $\left(\mathrm{VI}^{\circ}\right.$ nivel).

- Estudiantes de primer nivel de la educación secundaria $\left(\mathrm{VII}^{\circ}\right.$ nivel).

- Docentes de sexto grado de escuelas de educación primaria.

- Docentes de sétimo año de la educación secundaria.

- Padres, madres y encargados de los estudiantes y las estudiantes.

- Directores y directoras de instituciones educativas de educación secundaria.

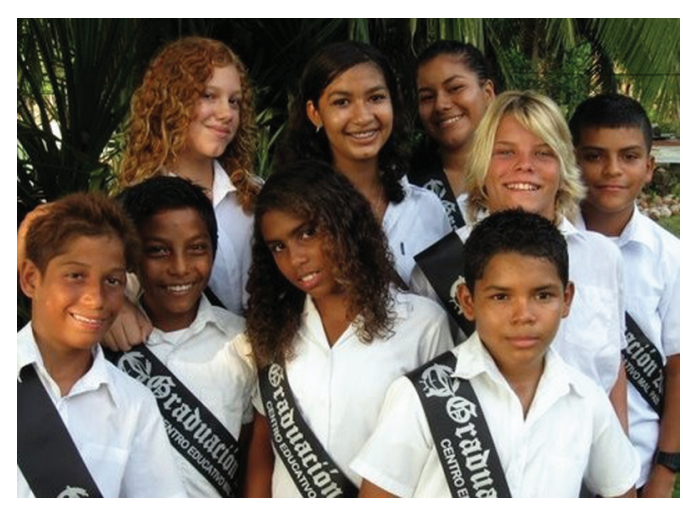

Los niños y las niñas que ingresan al colegio provienen de instituciones educativas de Educación Primaria, las cuales contrastan radicalmente con el modelo educativo prevaleciente en la secundaria.

Figura 4. Propiedad del autor.

\section{Conclusiones y principales hallazgos de la investigación}

De acuerdo con el estudio realizado, queda manifiesto que los cambios que experimentan los estudiantes en el proceso de primaria a secundaria son diversos; asociados a la diferencia de cultura que encuentra en la secundaria, se convierten en agentes que intervienen en el fracaso o éxito del proceso de transición de la primaria a la secundaria. Esta noción multifactorial de la problemática planteada es la que ha inspirado a concebir el abordaje de una propuesta que aporte ideas y mecanismos para propiciar y garantizar la permanencia de la juventud en la educación secundaria.

Cualquier iniciativa que atienda esta problemática debe centrarse en responder a las necesidades y demandas del estudiantado, de tal forma que cada docente desarrolle destrezas y habilidades que le permitan adecuar los contenidos y estrategias metodológicas en función de las particularidades de sus estudiantes, según lo requieran. Por consiguiente, se sugiere, fundamentalmente, cubrir las necesidades, carencias o problemáticas educativas particulares de los estudiantes que ingresan a la secundaria y así propiciar su permanencia. Al respecto se concluye: 
- A pesar de que al inicio de la investigación se concibió al estudiantado de sétimo año proveniente de zonas rurales como actor principal del estudio; conforme se avanzó se determinó que la problemática no solo afectaba a este grupo, sino que constituye una situación compartida que influye, de igual modo, en estudiantes de zonas semiurbanas y urbanas.

- La clave del éxito recae directamente sobre las instituciones de secundaria y las estrategias mediadoras que aplique el profesorado en sus aulas, tomando en cuenta el nivel de habilidad, contexto social y diversidad de sus estudiantes, como se plantea en la siguiente propuesta que constituye el producto esencial del estudio.

- El problema estudiado se centra en el primer año de la secundaria que no considera el modelo pedagógico de las escuelas de primaria del que proceden sus estudiantes, ni mucho menos el referido a las escuelas en contextos rurales.

- La carencia de procesos de articulación entre las prácticas metodológicas y elementos estructurales del sistema de la primaria hacia secundaria, inciden.

- La ausencia de inducción de primaria a secundaria promueve, en muchos casos, el abandono.

- Diversos condicionantes como: el tipo de institución, el horario de lecciones, el acceso al centro educativo y el ambiente de aula inciden en el desempeño académico del estudiantado.

- Urge la aplicación de estrategias de uso de una metodología adecuada, por parte de las docentes y los docentes de secundaria, que propicien el interés y mayor participación del estudiantado y que garanticen la permanencia y promoción.

- Existe la necesidad de implementar procesos de sensibilización e innovación pedagógica a docentes que llevan a cabo procesos de mediación en el sétimo año de la educación secundaria.

Algunos de los lineamientos y acciones que deben ser considerados para la implementación de una propuesta de la transición entre estos dos niveles y que atienda en forma pertinente la actual situación de vulnerabilidad del estudiantado que inicia la educación secundaria, se describen a continuación:

- Rediseño de los programas educativos dirigidos a la población que asiste a sétimo grado, con el propósito de establecer programas y metodología diferenciados, capaces de desarrollar ventajas dentro de cualquier ámbito y, al mismo tiempo, les permita a los jóvenes y las jóvenes continuar exitosamente en la educación secundaria.

- Actividades para el fomento de la autoconfianza en las capacidades y habilidades para afrontar nuevos retos por parte de cada estudiante.

- Integrar y comprometer a padres, madres y personas encargadas, en los procesos educativos que desarrollan sus hijos e hijas, así como brindarles la oportunidad de observar sus avances y logros en torno a su aprendizaje.

- Capacitar a los grupos docentes para el manejo de información útil que pueda ser interrelacionada, de manera pertinente, en el currículo para que, a partir de los temas del interés del estudiantado, puedan generarse diversos aprendizajes en las distintas áreas de conocimiento y destrezas.

- Jornadas de orientación a las familias acerca de la educación secundaria, la organización del centro y las formas más eficaces de apoyo a sus hijos e hijas.

- Talleres para docentes de sexto grado de la educación primaria y sétimo año de la educación secundaria para la socialización sobre las técnicas y metodologías de trabajo que realiza los(as) profesores (as) de primaria y viceversa. 
- Convivios entre estudiantes de sétimo y sexto grado para propiciar espacios de recreación y comunicación entre muchachos y muchachas de sexto y sétimo; en donde los muchachos puedan interactuar, lo cual les permitirá socializar sus experiencias como estudiantes sin temor de fracasar al llegar al colegio.

- Festivales artísticos y de expresión creativa en donde el estudiantado de primaria y secundaria participe en: coreografías, canto, retahílas, dibujo, disfraces, ejercicios dramáticos y otros; destacando la diversidad de culturas existentes en ambos grupos.

- Construir redes de difusión, promoción y distribución de información orientada al acceso a los derechos que deben gozar la juventud que ingresa a esta modalidad de enseñanza.

- Reconocer y valorar los conocimientos y habilidades de la población que inicia la educación secundaria, para integrarlos a los procesos educativos y reforzar la significancia de los mismos.

- Fomentar la participación de la juventud en la concepción, diseño, formulación, gestión y ejecución de proyectos educativos de interés comunitario que abarquen aspectos de índole cultural, deportivo, productivo y recreativo.

Estas acciones de apoyo intentan privilegiar al estudiantado, desde diversas áreas o aspectos propios de la vida en el colegio, como se plantea detalladamente en la siguiente propuesta producto del estudio.

\section{A. Justificación diagnóstica de la propuesta}

A partir del estudio mencionado se han identificado las siguientes situaciones que se considera importante resolver:

- Los docentes y las docentes que atienden sétimo año en instituciones de educación secundaria desconocen, en la mayoría de los casos, el perfil académico de ingreso de sus estudiantes, así como las particularidades del contexto del que provienen.

- Las capacitaciones y asesoramientos que se brindan al profesorado de los colegios no aportan lineamientos pertinentes relacionados con el tipo de mediación que debe ser implementada para propiciar el interés y permanencia de estudiantes que inician la educación secundaria.

- No existen criterios estandarizados que orienten, al cuerpo docente de secundaria, acerca del tipo de metodología que se debe implementar en el sétimo año de la educación secundaria, ni el tipo de atención que debe recibir el estudiantado procedente de escuelas rurales que inician este nivel de enseñanza.

- Se carece de políticas y acciones educativas oportunas que garanticen procesos de transición y articulación pertinentes entre los diversos ciclos y niveles de la Educación General Básica, con lo cual se provocan interferencias y obstáculos en el desarrollo de los procesos educativos en nivel regional y en el nacional.

- Se requiere asignar, a las instituciones educativas de secundaria, metas y propuestas claras, conducentes a la permanencia y promoción del estudiantado en el III $^{\circ}$ ciclo de la Enseñanza General Básica. 
- Urge la aplicación de estrategias concernientes a la concepción y uso de metodología adecuada por parte del personal docente de secundaria, que propicien el interés y mayor participación del estudiantado en los procesos pedagógicos, que garanticen la permanencia y promoción al inicio de la educación secundaria.

Por lo tanto, según las conclusiones del estudio, se destaca que no existe ni se aplica ningún tipo de estrategia pertinente que atienda adecuadamente la transición de la primaria a la secundaria. Con base en los problemas detectados, se propone la realización de un conjunto de acciones estratégicas que orienten procesos de transición para fortalecer y apoyar la gestión pedagógica de las docentes y los docentes que atienden poblaciones de estudiantes de sétimo año en instituciones de educación secundaria.

\section{B. Visión pronóstica}

Conforme a lo mencionado, para hacer frente a los problemas que afectan el desempeño académico y la permanencia en el primer año de la educación secundaria, se pretende, mediante la implementación de la presente propuesta, apoyar y orientar el trabajo pedagógico del grupo de docentes que atienden este nivel. De no atenderse tales problemas, se podría llegar a las siguientes situaciones:

- A corto plazo puede ocurrir que los índices de deserción y fracaso escolar, en el nivel de la secundaria, continúen agravando la actual situación educativa que muestra una mayor fragilidad en los primeros años de la secundaria.

- A mediano plazo se producirá mayor debilitamiento de la calidad de educación secundaria en zonas rurales y se aumentaría la brecha en el alcance de logros educativos entre zonas urbanas y rurales.

- A largo plazo se incrementaría la deserción en la población estudiantil de secundaria en todos sus niveles. Lo anterior, como consecuencia del bajo rendimiento debido a las bases cognitivas que los estudiantes traen de los niveles básicos de la secundaria. Del mismo modo, se estaría condenando, a un gran segmento de esta población, a la exclusión educativa.

\section{Una respuesta pertinente que propicie la permanencia del estudiantado}

\section{Génesis de la propuesta}

La Propuesta: Lineamientos orientadores para el desarrollo de procesos de transición de la primaria a la secundaria que contribuyan a la permanencia de los estudiantes de sétimo año en la educación secundaria se sustenta en los hallazgos, aportes y resultados obtenidos de la consulta realizada a estudiantes sexto y sétimo año, docentes de primaria y secundaria, así como directores y directoras de dichas instituciones, en el marco de la investigación denominada: Mejoramiento del desempeño académico en el sétimo año de la educación secundaria, en los estudiantes y las 
estudiantes provenientes de las escuelas rurales, como una forma de propiciar su permanencia en la educación secundaria (Miranda, 2010). Asimismo constituye el producto principal generado a partir de dicho estudio. Describe y propone acciones pertinentes para todo cuerpo docente que atiende la mediación pedagógica con estudiantes de primer ingreso a la educación secundaria. Constituye herramienta para orientar y potenciar el tratamiento pedagógico de este grupo en particular.

\section{Objetivos de la propuesta}

1. Proponer a directores, directoras y docentes de sétimo año, un conjunto de lineamientos orientadores de procesos de transición de la primaria a la secundaria que contribuyan a la permanencia de los estudiantes en la educación secundaria.

2. Fortalecer la práctica pedagógica de docentes de sétimo año mediante estrategias y acciones metodológicas orientadas al fortalecimiento de la expresión y comunicación del estudiantado durante el transcurso del sétimo año.

3. Brindar a docentes de sétimo año un conjunto de estrategias dirigidas al uso y aplicación de metodología participativa que fortalezca la autoestima y el desempeño académico del estudiantado que inicia la educación secundaria.

4. Involucrar a las familias de las estudiantes y los estudiantes en los procesos formativos mediante actividades que propicien la integración y el vínculo colegio-hogar.

5. Propiciar la permanencia del estudiantado en el III $^{\circ}$ Ciclo de la Enseñanza General Básica.

\section{E. Logros y beneficiarios}

Los alcances y propuesta resultantes de este estudio aportarán beneficios a la población estudiantil de instituciones de educación secundaria, así como al su profesorado, específicamente el que atiende la población de sétimo año. Del mismo modo, sus comunidades obtendrán beneficios posteriores y, consecuentemente, todos los usuarios del Sistema Educativo Costarricense que conforman las comunidades educativas rurales y urbanas del país.

\section{F. Estructura y descripción de la propuesta}

La propuesta está conformada por los siguientes componentes:

1. Justificación de la propuesta

2. Áreas estratégicas de la propuesta

3. Estructura de gestión y de recursos

4. Condiciones básicas para la ejecución

5. Recursos requeridos para la implementación de la propuesta en cada colegio

6. Evaluación del impacto 


\section{Justificación de la propuesta}

Como mandato de la Educación Costarricense, la permanencia de la población adolescente en el sistema educativo debe estar implícita como una prioridad en la misión, visión y propósitos de cada institución, acorde con el contexto situacional y valores que sustentan su filosofía, estrategias y objetivos que definan la forma de trabajo de cada institución, ya que garantizar el derecho a la educación obligatoria desde cada colegio requiere actuaciones coordinadas entre los actores del contexto institucional y la consolidación de procesos y etapas sistemáticas.

La propuesta pretende, entre otros fines, lograr la integración de los actores que conforman el entorno social de los colegios. Por la riqueza del contexto social en el que los mismos están inmersos, se hace imprescindible valorar su potencial y establecer múltiples alianzas de apoyo y propiciar la participación de personas y organizaciones que se relacionen directa o indirectamente con estas instituciones educativas. Esta condición ideal debe entenderse como un vínculo imprescindible entre colegio-comunidad, en donde la institución educativa debe favorecer, en primera instancia, la dimensión pedagógica que es su razón de ser.

\section{2. Áreas estratégicas de la propuesta}

El conjunto de procedimientos y acciones que desarrollan profesoras y profesores del primer nivel de la secundaria se puede establecer en tres grandes dimensiones o áreas de acción: área académica, área social y área administrativa (figura 1). Asimismo, la naturaleza de estas instituciones permite y requiere, en procura de afianzar las bases cognitivas del estudiantado y garantizar así su permanencia, la integración coordinada de estas áreas para alcanzar objetivos integrales que beneficien a la comunidad estudiantil, así como facilitar y apoyar la labor que desarrollan el personal docente de sétimo año.

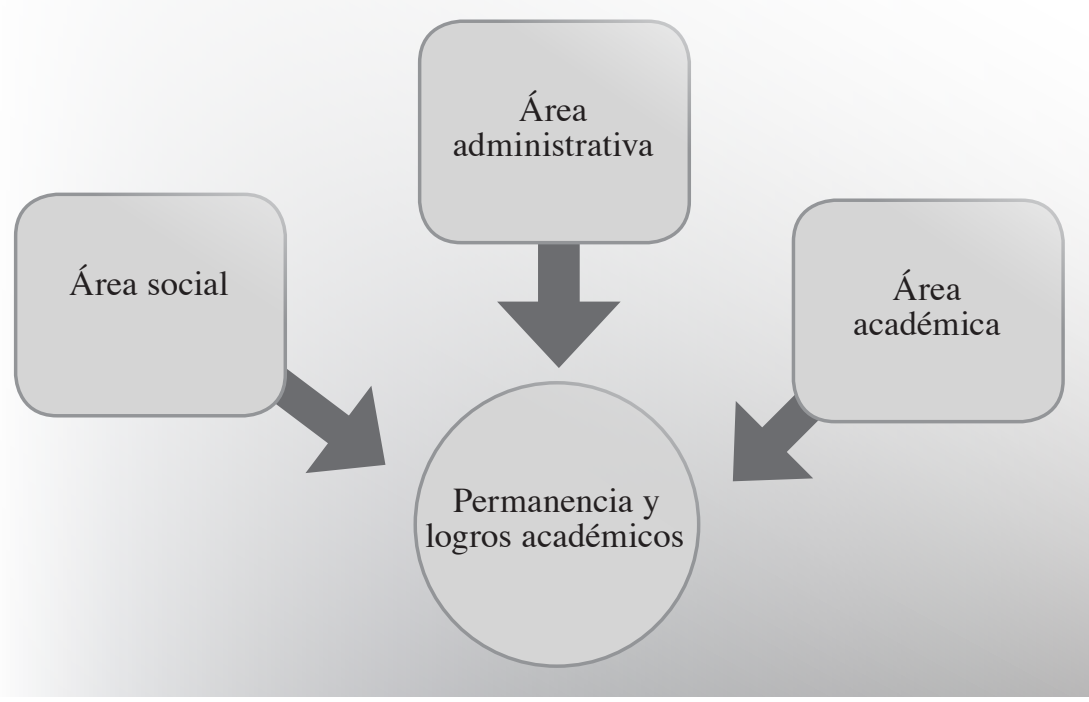

Figura 1: Áreas estratégicas de la propuesta. 
Cada una de las áreas de esta propuesta debe interactuar y estar intrínsecamente vinculada con las demás. Razón por la cual resulta indispensable considerar cada una de las mismas, como espacios de incidencia presentes en los colegios. Estas deben ser reforzadas, ya que manifiestan una alta influencia en el alcance de logros educativos, no solo en lo interno de la institución, sino además en su proyección social, función implícita de las instituciones de educación secundaria. Por lo tanto, la propuesta está constituida por la diversa gama de interrelaciones que se dan continuamente entre estas tres áreas.

\section{Área académica}

Debe estar conformada por la totalidad de los elementos relacionados con los procesos pedagógicos que se desarrollan en las instituciones de educación secundaria, debe asumir el tratamiento de los contenidos y la metodología que aporte el mayor número de logros en el aprendizaje del estudiantado.

El grupo de docentes de sétimo año debe enfrentar, al inicio de cada curso lectivo, la atención de estudiantes que presentan carencias en cuanto a hábitos de estudio, apoyo familiar, organización del tiempo y del espacio, participación activa en el desarrollo de las lecciones y acatamiento de instrucciones, entre otros aspectos. Esta atención requiere de una especial disposición del docente para encauzar y motivar a sus estudiantes a incorporar lo que corresponda y superar los retos que algunos representan. Desde esta óptica, las docentes y los docentes deben apropiarse de una concepción más adecuada de la enseñanza, que reconozca y privilegie los diversos tipos de inteligencia y estilos de aprendizaje, la relación del conocimiento con la realidad y las vivencias de sus estudiantes, así como la naturaleza social y emocional que les caracteriza en estas edades.

Algunas prácticas que se consideran fundamentales para favorecer la transición o el paso equilibrado entre los niveles de primaria y secundaria, se plantean a continuación:

- Valoración de los resultados de las pruebas de diagnóstico (perfil de entrada) y evaluaciones iniciales en cada asignatura, para la toma de decisiones pedagógicas y propuestas que atiendan, de forma planificada, las necesidades cognitivas que requiere el estudiantado procedente de sexto grado, como plataforma básica para su buen desempeño en el inicio y resto de la educación secundaria. En este sentido, los procesos evaluativos, como aspectos sustanciales para el aprendizaje y como medios proveedores de información, deben ser destinados a servir de base para la toma de decisiones; esto por cuanto en el nivel de sétimo año se requiere ofrecer un mayor acompañamiento, tanto en los procesos cognitivos como en los evaluativos.

- Creación de espacios de aprendizaje significativos y aprovechamiento de las herramientas tecnológicas para la construcción efectiva del conocimiento, mediante métodos y técnicas atractivas y motivadoras, aprovechando, creativamente, las tecnologías y recursos disponibles de la institución y promoviendo la participación, tanto de estudiantes, de padres y madres, familias y vecinos, para incrementar y optimizar los medios destinados para la enseñanza y el aprendizaje.

- Fortalecimiento de la comprensión y expresión del estudiantado de sétimo año desde todas las asignaturas, mediante el desarrollo de las capacidades y habilidades que se consideran básicas e imprescindibles para que puedan continuar aprendiendo, pero además vistas como herramientas para la vida, promoviendo así la autodisciplina y la autoorganización como habilidades para 
la vida, condiciones que son indispensables en el desarrollo de una personalidad autónoma e interdependiente, lo cual también se revierte, positivamente, en los diversos aspectos académicos.

- Implementación de clases y experiencias innovadoras de aprendizaje en las que se promueva el trabajo en equipo y se favorezca la motivación, la valoración, la inclusión, la integración, la complementariedad de capacidades, habilidades y conocimientos; así como la adaptabilidad y la socialización que propicien una mejor convivencia y disciplina, individual y grupal.

- Acompañamiento y tutoría académica a cada estudiante mediante el seguimiento y monitoreo sistemático de su itinerario educativo, rendimiento académico y ritmo de aprendizaje, con el propósito de inducir y orientar la adopción de hábitos y técnicas de estudio adecuadas a las nuevas exigencias académicas que les plantea la secundaria. En la medida que adquieran buenos hábitos de trabajo y estudio desde el primer año, se facilitará su paso por esta etapa educativa en la que el esfuerzo debe ser mayor en contraste con la educación primaria.

- Elaboración conjunta de un manual institucional sobre: técnicas de estudio, modalidades de aprendizaje, trabajos en grupo, planificación de labores académicas, entre otros. Debe ser elaborado con la participación activa de docentes y estudiantes, con carácter orientador, que describa y recomiende al estudiantado, los nuevos requerimientos académicos que deben asumir y los parámetros a los que deben ajustarse. En este sentido, es necesario llegar al consenso, entre docentes, acerca de qué trabajos y actividades deben ser estandarizadas y compartidas entre ellos, para facilitar el desempeño y avance progresivo y gradual de sus estudiantes.

- Interacción formativa entre docentes y estudiantes, que instaure, en la convivencia cotidiana, un clima de respeto, comprensión y aceptación del estudiante y en el que los docentes manifiesten apertura mental y conciencia de la diversidad y multiculturalidad estudiantil con la que trabajan.

- Adopción de estrategias de evaluación de los aprendizajes concordantes con las particularidades de cada asignatura y haciendo uso de diferentes formas de evaluación de acuerdo con el perfil de entrada y las necesidades educativas del estudiantado que ingresa al sétimo año.

- Coordinación, entre docentes, para el abordaje del estudio integrado de los contenidos, mediante procedimientos compartidos y articulados entre las diversas asignaturas, De esta forma se apoya y se reafirma el aprendizaje desde distintas disciplinas y metodologías; además, se evita la errada concepción de materias difíciles y materias fáciles que conciben el conocimiento en forma fragmentada y dificultan el aprendizaje integral.

- Incremento y reforzamiento de las medidas de apoyo al alumnado con dificultades. Esto supone medios para poder organizar procesos flexibles que involucren al profesorado en la atención pedagógica que requiere el estudiantado con rezago educativo, debido a factores como: una pobre formación en la primaria, inseguridad y baja autoestima, ausentismo involuntario y dificultades de acceso, entre otras.

\section{Área social}

Representa un área determinante en el alcance de logros, ya que constituye el entorno social común e inmediato de estas instituciones y sus estudiantes. Se caracteriza por la vasta interacción entre todas las personas vinculadas al estudiantado, que al mismo tiempo se benefician directa e indirectamente de la institución educativa. 
Es imprescindible concebir a las niñas, niños y adolescentes que ingresan al colegio, como personas en formación, con diversas situaciones emocionales debidas a los cambios propios de la adolescencia y sus implicaciones en la autonomía y autoestima.

En esta etapa del desarrollo, los niños y niñas provenientes de la primaria y que ingresan a la secundaria deben encontrar, en el colegio, un espacio que les permita sentirse valorados como personas, así como recibir buen trato y amistad de quienes serán sus formadores y formadoras.

En muchos casos, esta condición es medular, por encima del aprender, ya que logra mantener el interés, motivación, compromiso y permanencia del estudiantado. De aquí la trascendencia en conformar espacios para la interacción social y optimizar la calidad de las relaciones que se establecen en los colegios.

Se citan a continuación las tres estrategias que pueden potenciar el área social:

- Integración de las familias del estudiantado en la vida escolar, donde asuman su responsabilidad de participar en actividades que se relacionen con la educación de sus hijos e hijas, brindándoles apoyo y fomentando la comunicación asertiva.

El hecho de que los niños, niñas y adolecentes se ajusten adecuadamente a estos cambios que impone la secundaria puede ser determinante en el éxito o fracaso escolar durante esta etapa educativa y, consecuentemente, en el posterior paso del estudiante a niveles superiores. Por lo tanto, el papel que deben desempeñar las familias durante el primer año de la secundaria no debe estar relegado a la simple observación, sino que debe ser un rol participativo y activo de modo que facilite, dentro de la independencia y autonomía que se le debe dar al alumno en estas edades, su adaptación e integración tanto social como académica.

El balance entre estas dos dimensiones del contexto escolar es sustancial como un marco de apoyo idóneo para fortalecimiento de la personalidad y autoestima de cada estudiante.

Se sugiere que padres, madres y encargados apoyen, desde el hogar, la labor docente y refuercen la autonomía y desempeño de sus hijas e hijos mediante:

- Contacto permanente, con las docentes y los docentes de sus hijas e hijos.

- Valoración de sus hijos e hijas en sus aspectos positivos y en todos sus esfuerzos.

- Animar constantemente el desempeño académico de sus hijas e hijos en procura de mayores logros educativos.

- Evitar los premios y castigos asociados a las responsabilidades académicas que deben asumir sus hijos durante sus estudios.

- Establecimiento conjunto de acuerdos, compromisos y metas viables ante bajas calificaciones escolares obtenidas, entre encargados e hijos

- Convencimiento absoluto y compartido en la familia, acerca de la importancia del estudio y la formación de sus hijas e hijos.

- Mostrar interés por los temas que estudian.

- Establecimiento de un sitio adecuado para el estudio y sus respectivos horarios.

- Evitar la descalificación de las profesoras y profesores y apoyar la labor de los mismos desde el hogar. 
- Proyectos extracurriculares que integren al colegio con la comunidad mediante actividades complementarias de la formación del estudiantado, en aspectos relacionados con su cultura, el desarrollo de experiencias artísticas y deportivas. Del mismo modo se pueden desarrollar proyectos que vinculen la vida del colegio con la realidad y las necesidades de la comunidad educativa.

- Encuentros y convivencias intra e interinstitucionales, en los que se dé prioridad al desarrollo de destrezas y habilidades comunicativas y de convivencia entre estudiantes de sétimo año y niveles superiores, mediante experiencias culturales, deportivas y artísticas compartidas, que potencien el desarrollo personal, fortalezcan la autoestima y consoliden el establecimiento de lazos sociales.

\section{Área administrativa}

Las estrategias y acciones que plantea la propuesta desde el área administrativa son de vital importancia, debido a que constituyen el motor en la toma de decisiones y la ejecución oportuna de las estrategias que garanticen, al centro educativo, la eficiencia de los procesos que se lleven a cabo en beneficio de cada comunidad educativa.

El área administrativa debe responder, de modo eficiente, a las necesidades y expectativas del alumnado, disponiendo de espacios propicios para el aprendizaje, recursos bibliográficos existentes y utilizables por las los estudiantes y los estudiantes, para que puedan hacer uso adecuado y así apoyar su propio crecimiento académico. Asimismo, mediante la planificación y ejecución se deben concretar las estrategias planteadas en las otras áreas de la propuesta. Dentro de esta dimensión se sugieren las siguientes actividades:

- Sesiones previas (año anterior) con carácter orientador en las que se informe a los padres, madres y encargados de estudiantes de $6^{\circ}$ de la educación primaria sobre el tema: Adolescencia y nuevos retos educativos a partir del inicio de la educación secundaria.

- Jornadas de orientación a las familias acerca de la educación secundaria, la organización del centro, el compromiso como padres y madres, así como las formas eficaces de apoyo al alumnado y relación con el centro.

- Diseño y consolidación de proyectos educativos (culturales, artísticos, deportivos) que trasvasen los límites de los niveles de la secundaria para propiciar el interés, compromiso y sentido de pertenencia del estudiantado de $7^{\circ}$ año con su grupo de docentes, compañeros y el quehacer de su colegio.

- Coordinación entre el profesorado de primaria y secundaria para el establecimiento de medidas y criterios comunes en la secuenciación de los contenidos, metodología y criterios de evaluación, sobre todo en las áreas que requieren dominio acumulativo para los años posteriores, como es el caso de Español y Matemática. A partir de esta noción se sugieren las siguientes acciones: 
- Proyectos educativos que articulen de trabajo académico que se lleva a cabo en el último año de las escuelas y en el primer año de la secundaria.

- Proyectos educativos que traspasen de sétimo año a octavo año la secundaria.

- Reuniones de coordinación con docentes de sexto y sétimo año.

- Evaluación permanente de los procesos pedagógicos.

- Adopción de metodologías dinámicas y activas que propicien el interés y compromiso en el estudiantado de sétimo año.

- Coordinación de reuniones con miembros de las familias del estudiantado para informar acerca del avance y logros académicos de sus hijas e hijos.

- Conformación de un clima escolar propicio que favorezca la colaboración entre el profesorado y un ambiente de exigencia académica, y aceptación y ayuda a las estudiantes y los estudiantes. Implica la creación de un ambiente ideal de centro, en concordancia con las necesidades y expectativas de los estudiantes, que les proporcione tranquilidad, seguridad y sentido de pertenencia con su institución. Esto implica un compromiso de la gestión directiva del colegio, del equipo docente, del estudiantado y de sus respectivas familias.

- Planificación, ejecución y evaluación de los procesos educativos en sétimo año de acuerdo con la problemática que caracteriza la adolescencia, edades en que cursan este nivel.

- Jornadas de orientación y formación a las familias con temas propios que inciden en la educación secundaria durante la adolescencia, la organización del centro educativo, las formas eficaces de apoyo al alumnado y la relación con el centro.

- Ejecución de propuestas dirigidas al mejoramiento de la práctica pedagógica de docentes que contribuyan a la permanencia de estudiantes en el sétimo año de la educación secundaria.

- Cumplimiento de la atención de necesidades educativas de los estudiantes y las estudiantes, así como los mecanismos necesarios para que el profesorado conozca los diferentes tipos de adecuaciones curriculares y los más recientes lineamientos nacionales para atender a estudiantes con discapacidad o necesidades educativas de toda clase.

- Seguimiento y control riguroso de ausentismo en forma continua y permanente, con el propósito de localizar y recuperar al alumnado que falta con frecuencia a clases o dejan de asistir a la institución sin ninguna justificación o motivo comunicado.

- Coordinación de los servicios internos de la institución tales como: Orientación, Comités de apoyo y psicología, así como el establecimiento y contacto permanente con instituciones y servicios externos: Patronato Nacional de la Infancia (PANI), Instituto sobre Alcoholismo y Farmacodependencia (IAFA) y el Consejo de la Persona Joven, para la implantación y generalización de la presente propuesta.

- Desarrollo de procesos de actualización permanente y capacitaciones dirigidas a docentes de sétimo año, que les permita incluir, en su práctica pedagógica, innovaciones científicotecnológicas y metodológicas relacionadas con su disciplina. Con ello se pretende enriquecer 
su labor y, consecuentemente, estimular el entusiasmo y la participación interactiva del estudiantado.

\section{Estructura de gestión y recursos}

Para la ejecución de la propuesta, se tomará en cuenta el factor tiempo como recurso indispensable a lo largo del curso lectivo, el cuerpo docente de sétimo año, potenciado con el trabajo en equipo, así como los recursos físicos-tecnológicos y de comunicación con que dispone cada institución educativa de secundaria.

\section{Condiciones básicas para la ejecución}

Para poner en práctica la propuesta en instituciones de educación secundaria es necesario establecer en consenso sobre la necesidad de mejorar y fortalecer el tipo de labor educativa que ahí se desarrolla. Para esto se requiere la participación integrada de cada uno de los elementos que conforman las áreas o dimensiones, como se plantea en la presente propuesta. Resulta claro que la incorporación en la vida del colegio de las personas encargadas de las niñas y niños que acceden al sétimo nivel debe inspirar la concepción de metodología proactiva que logre aprovechar las aptitudes innatas de los miembros de las familias, sus interacciones y sus potencialidades en cuanto al conocimiento de su entorno social.

Como requisito indispensable, se debe contar con un equipo de trabajo colaborador que apoye la gestión administrativa de maestras y maestros, de manera que las responsabilidades de estas docentes y estos docentes se concentren en los procesos pedagógicos. Cada una de las estrategias propuestas en cada una de las áreas debe ser entendida como una actividad permanente antes y a lo largo de cada curso lectivo, lo cual implica su articulación constante con el cronograma de cada institución.

Se considera imprescindible contar con la colaboración de maestros y maestras de sexto grado que brinden sus testimonios y criterios relacionados con su desempeño con el estudiantado que eventualmente ingresará a la secundaria. Asimismo, se considera de gran valor la participación de los miembros de la comunidad educativa, tal es el caso de padres y madres de familia, personas egresadas, vecinos y población estudiantil de niveles superiores.

\section{Recursos requeridos para la implementación de la propuesta en cada colegio}

Se requiere contar con un conjunto de recursos básicos que garanticen la consecución de las actividades establecidas. Para ello se sugieren cuatro tipos de recursos que deben se aprovechados integralmente como se muestra en la figura 2. 
URL: http://www.una.ac.cr/educare

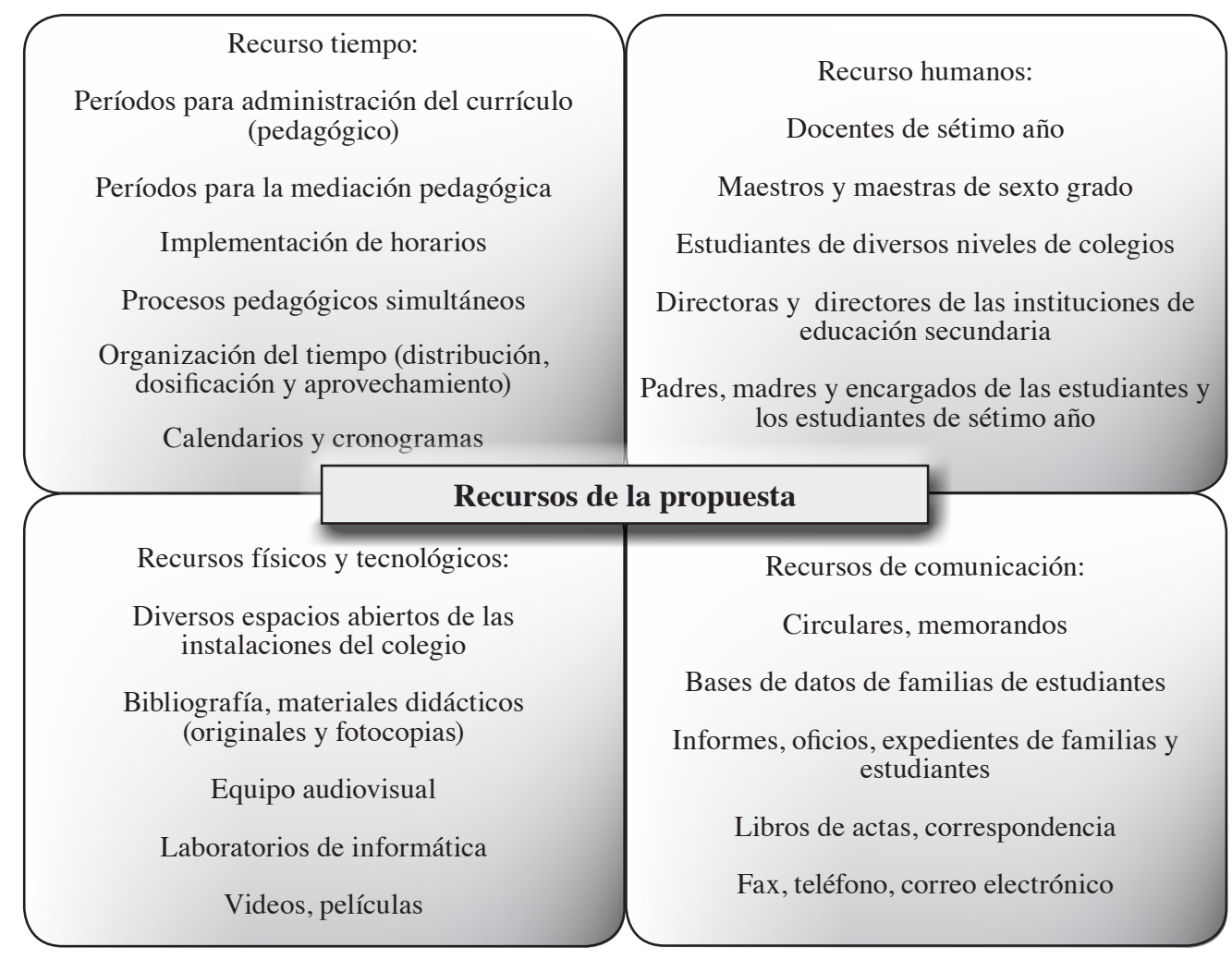

Figura 2: Recursos de la propuesta (elaboración propia a partir del estudio).

\section{Evaluación del impacto}

El impacto de las acciones ejecutadas se evaluará luego de implementarse por un periodo de nueve meses, antes del cierre de cada curso lectivo, a través de la realización de talleres. Lo anterior obedece al propósito de determinar si las acciones que se definieron en la propuesta, se llevan a cabo y si las mismas cumplen con los objetivos propuestos.

Con base en los criterios y opiniones que los participantes señalen, se realizarán las modificaciones y ajustes que se requieran para dar el acabado final y enriquecer la propuesta con sus aportes. Asimismo, el cronograma que implemente las acciones deberá estar conformado por un conjunto de actividades distribuidas antes, durante y después del curso lectivo en el sétimo año. El mismo debe obedecer a las particularidades y condiciones propias de cada institución, además debe establecer los parámetros con los que se abordará la ejecución, desarrollo y evaluación de los resultados y productos de las actividades que plantea la propuesta.

La mejor estrategia de evaluación que se debe asumir en los colegios, en relación con el presente planteamiento, es concebir el alcance de logros académicos de las estudiantes y los estudiantes y su permanencia a lo largo del $\mathrm{III}^{\circ} \mathrm{Ciclo}$, como fieles indicadores de su verdadero impacto y pertinencia en cada una de las instituciones de educación secundaria y, posteriormente, en las respectivas comunidades donde se encuentran insertas. 


\section{Referencias bibliográficas}

Alfaro, M., Brenes, A., Gamboa, A., Jiménez, S., Martín, J., Ramírez, A. ... Zúñiga, A. (2008). Construcción del perfil de desempeño docente para el sétimo año de la Educación Pública (Informe final de investigación del Proyecto Perfiles, Dinámicas y Desafíos de la Educación Costarricense, tercera etapa). Heredia, Costa Rica: CIDE-Universidad Nacional.

Consejo Nacional de Política Pública de la Persona Joven. (2008). Primera encuesta nacional de Juventud Costa Rica (principales resultados). San José, Costa Rica: Fondo de Población de las Naciones Unidas (UNFPA). Recuperado de http:/www.unfpa.or.cr/dmdocuments/ Encuesta Juventud.pdf

Guendel, L., Osorio, R. y González, M. (1999). La educación: política social para el ejercicio de la ciudadanía. En Política Social y Educación en Costa Rica (pp. 21-57). San José, Costa Rica: UNICEF-Costa Rica.

Hernández, A., León, A., Ruiz, S., y Zúñiga, I. (2002). Validación de un módulo de selección de candidatos a docentes, fundamentado en la idoneidad de sus características personales y la percepción de su futuro papel profesional. San José, Costa Rica: Coordinadora Educativa Cultural Centroamericana (CECC).

Miranda, A. (2008). Juventud rural y educación: Un desafío a la equidad (Cap. 6). En M. Gutiérrez y N. Piedra (Coords.), VI Estado de los derechos de la niñez y la adolescencia en Costa Rica (pp. 177-186). San José, Costa Rica: UNICEF. Reecuperado de http://www.unicef.org/costarica/13222.htm

Miranda, A., Bonilla, N. (2010). Mejoramiento del desempeño académico en el sétimo año de la Educación Secundaria, en los estudiantes y las estudiantes provenientes de las escuelas rurales, como una forma de propiciar su permanencia en la Educación Secundaria. (Tesis de Maestría sin publicar). Universidad Nacional de Costa Rica, Heredia.

Programa Estado de la Nación. (2011). Estado de la Educación 3 (Cap. 3). San José, Costa Rica: Autor. Recuperado de http://www.estadonacion.or.cr/images/stories/informes/educacion_003/ docs/Parte 1 Capitulo 3.pdf 